\title{
Effect of Vitamin D Injection in Recurrent Benign Paroxysmal Positional Vertigo with Vitamin D Deficiency
}

\author{
Gu II Rhim ${ }^{10}$ \\ ${ }^{1}$ The One Otorhinolaryngology Clinic, Paju, South Korea \\ Int Arch Otorhinolaryngol 2020;24(4):e423-e428.
}

\begin{abstract}
Address for correspondence Gu II Rhim, MD, PhD, The One Otorhinolaryngology Clinic, 2 sicheong-ro, Paju 10924, Korea (e-mail: guzi9170@naver.com).
\end{abstract}

\begin{abstract}
Keywords

- benign paroxysmal positional vertigo

- vitamin D deficiency

- recurrence

Introduction There have been reports indicating that patients with frequently recurring benign paroxysmal positional vertigo (BPPV) had vitamin D deficiency, and some studies indicated that the treatment of severe vitamin $D$ deficiency is effective in the reduction of the recurrence of BPPV.

Objective The purpose of the present study was to examine the effects of Vitamin $D_{3}$ injection on recurrence among patients with a $10 \mathrm{ng} / \mathrm{mL}$ or lower 25 -hydroxyvitamin $D$ blood concentration diagnosed with BPPV.

Methods Among 99 patients with idiopathic BPPV with vitamin D deficiency, 25 patients (case group) were submitted to 3 to 4 injections of 200,000 IU of vitamin $D_{3}$ in the first year. In total, 50 patients in the control group were selected through frequency matching, with 25 patients in the case group. Age, gender, and type of BPPV are used in matching variables with 1:2 matched data. The subjects of the study group were followed up for 24 months.

Results The differences in relapse rates between the case and the control groups were examined using the non-parametric Kruskal-Wallis test for $k$ independent samples. With regard to the relapse rates of the entire case and control groups by period, from 0 to 6 months $(p<0.531)$, from 7 to 12 months $(p<1.000)$, and from 13 to 24 months $(p<0.711)$, and in the entire study period $(p<0.883)$ there were no statistically significant differences.

Conclusion The present case-control study indicated that vitamin $\mathrm{D}_{3}$ injection had no significant effect on the recurrence of BPPV patients with vitamin D deficiency when age, gender, and type of BPPV were homogeneous between the two groups.
\end{abstract}

\section{Introduction}

Benign paroxysmal positional vertigo (BPPV) is a disorder arising in the inner ear, in which otoconia enter the semicircular canal or adhere to the cupula and cause clinical symptoms due to changes in position.

The particle repositioning maneuver is known to be the standard treatment, but the 1 -year recurrence rate is $\sim 20 \%$, and recurrence rates within 4 to 5 years are of $\sim 40 \%$ to $50 \%{ }^{1-3}$
Some studies indicated that vitamin D is involved in the metabolic process of calcium in the vestibular organ, and vitamin D deficiency affects the development of BPPV. ${ }^{4,5}$

Otoconia are biocrystals that couple mechanic forces to the sensory hair cells of the vestibular system. Otoconia are composed of calcium carbonate $\left(\mathrm{CaCO}_{3}\right)$ crystals and glycoproteins, and are connected with protein fibers on hair cells. ${ }^{4}$ Little has been done on issues related to otoconia regeneration and degeneration in humans. But the generation, growth, and received

November 10, 2018

accepted

October 10, 2019
DOI https://doi.org/

10.1055/s-0039-3402431. ISSN 1809-9777.
Copyright $(2020$ by Thieme Revinter

Publicações Ltda, Rio de Janeiro, Brazil
License terms

(요 (1) $\Theta \circledast$ 
degeneration processes of otoconia are known to be regulated by the calcium metabolic process of the vestibular organ in animal studies. ${ }^{6}$ Some study results indicated that vitamin D-related epithelial calcium channel proteins as players for otoconia formation are involved in determining the crystalline morphology, growth, and stability of otoconia. ${ }^{7}$

Recent studies ${ }^{8-10}$ indicated that osteoporosis, which is an example of calcium metabolic disease, is associated with the development and recurrence of BPPV. There have also been reports indicating that patients with frequently recurring BPPV had vitamin D deficiency, along with study results indicating that low levels of vitamin $\mathrm{D}$ were related to the development of BPPV, while very low levels were associated with the recurrence of BPPV. ${ }^{11-13}$ Moreover, a study ${ }^{14}$ indicated that the treatment of severe vitamin D deficiency is effective to reduce the recurrence of BPPV.

The present study was conducted to examine the effect of vitamin $D_{3}$ injection on BPPV recurrence in vitamin D-deficient patients diagnosed with idiopathic BPPV who had 25-hydroxyvitamin D (25-OH vitamin D) blood concentrations $<10 \mathrm{ng} / \mathrm{mL}$.

\section{Materials and Methods}

The present study was conducted at a primary otorhinolaryngology clinic. The subjects of present study were selected from among 299 patients diagnosed with idiopathic BPPV with vitamin D deficiency from June 2014 to February 2016 at our outpatient clinic, and, among those, 99 individuals (23 males, 76 females; age range: $15-67$ years) were recruited. In accordance with the Declaration of Helsinki, oral and written informed consent about the design, aim, and clinical implication of our study was obtained from all of the participants.

A total of 299 subjects with BPPV were examined to select the study group. Patients with renal disease, Ménière disease, or any ear disease, such as chronic otitis media, vestibular neuritis, and otitis media on the same side accompanying BPPV from the beginning, as well as patients with secondary BPPV were excluded. Patient information on age, gender, comorbidities (diabetes, hypertension, and hyperlipidemia), 25-OH vitamin D concentrations and the type and location of the semicircular canals involved in the initial BPPV attacks were reviewed retrospectively. The subjects were instructed to return to the clinic immediately if they suspected BPPV recurrence, and direct patient telephone calls were made to ensure the accuracy of the recurrence data. The entire observation period was of 26 months; The first 2 months were the period of waiting for $25-\mathrm{OH}$ vitamin $\mathrm{D}$ levels in the blood to be normal, and the remaining 24 months were follow-up periods (-Fig. 1).

Blood samples were collected on the first visit to the hospital and sent for tests on the same day. The 25-OH vitamin D concentrations of the patients were measured using a chemiluminescence immunoassay (CIA; Centaur, Siemens, Munich, Germany) as a serum test. Levels lower than $10 \mathrm{ng} / \mathrm{mL}$ were accepted as vitamin D deficient. In the case group, the 25-OH vitamin D concentration was re-evaluated 12 months after the initial diagnosis.

Among the 99 patients with idiopathic BPPV with vitamin D deficiency, 25 were enrolled in the present study. During the follow-up period of 2 years, 25 patients (the case group) were injected with $200,000 \mathrm{IU}$ of vitamin $\mathrm{D}_{3}$ through an intramuscular injection into the buttocks within 2 weeks after the initial diagnosis. Twenty patients were submitted to 200,000 IU of vitamin $D_{3}$ every 3 months after that, and 5 patients were submitted to 200,000 IU of vitamin $D_{3}$ every 4 months. In total, 3 to 4 injections were administered in the first year. The vitamin D injection solution used was in 1-ml ampoules containing 200,000 IU (5mg) of cholecalciferol (Merit D, Huons Pharmaceutical Co., Seongnam, Gyeonggi, South Korea). The 25-OH vitamin D blood test was conducted again at the first year of follow-up after the initial diagnosis. The case group was divided into 2 subgroups: subgroup I included 10 subjects diagnosed with posterior canal BPPV and lateral canal BPPV, and subgroup II included 15 subjects diagnosed with cupulolithiasis BPPV and multiple BPPV.

Among the 99 patients with idiopathic BPPV with vitamin D deficiency examined, 50 patients in the control group were selected through frequency matching with 25 patients in the case group.

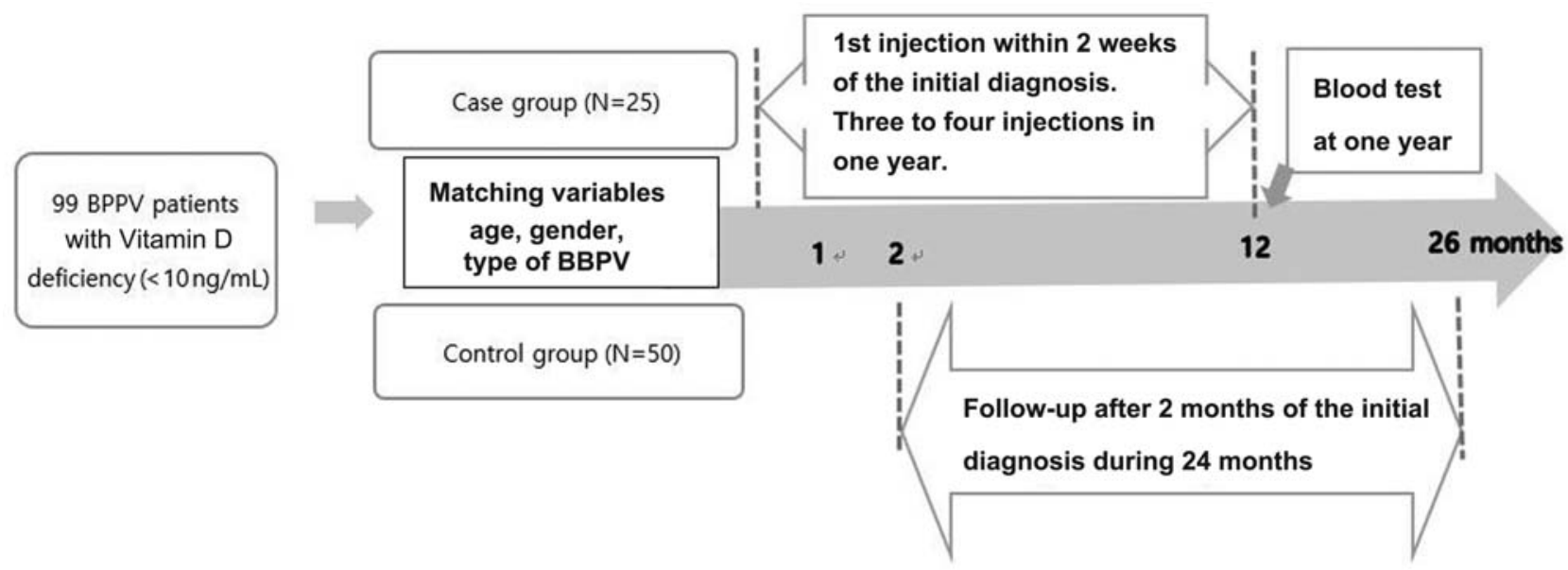

Fig. 1 Study design. 
The age variable used in matching is a confounding variable that is causally related to the occurrence and recurrence of $\mathrm{BPPV}$, and it also has the nature of an effect modifier. An effect modifier refers to a confounding variable that causes different degrees of disturbance depending on its layers. Age can be said to act as a confounding variable and as an effect modifier. Gender is also a variable is related to the occurrence and recurrence of BPPV. Recurrence affected especially postmenopausal women due to the changes caused by sex hormones. And canal types of BPPV are known to be related with recurrence. Therefore, age, gender, and type of BPPV are used in matching variables, which were controlled using statistical analysis with 1:2 matched data. The control group was divided into 2 subgroups: subgroup III included 21 subjects diagnosed with posterior canal BPPV and lateral canal BPPV, and subgroup IV included 29 subjects diagnosed with cupulolithiasis BPPV and multiple BPPV.

All patients underwent physical examinations using pure tone audiometry, videonystagmography, and video Frenzel goggles (SLMED Co., Seoul, South Korea) when they first visited the hospital and were diagnosed and treated by a physician. The Dix-Hallpike, supine roll, and supine straight head hanging tests were performed to determine the location of the lesions. ${ }^{13}$ For posterior semicircular canal BPPV, the Dix-Hallpike test was positive if nystagmus was recorded with appropriate positioning, latency, duration, and fatigability. Lateral semicircular canal BPPV (LC-BPPV) was diagnosed by positional nystagmus changing in the horizontal direction concurrent with vertigo triggered by the supine roll test. According to the direction of the nystagmus as horizontal geotropic and apogeotropic nystagmus, the LC-BPPV was classified as canalolithiasis or cupulolithiasis respectively. Multiple-canal BPPV includes either involvement of the same canal on both sides, or simultaneous involvement of different canals on the same or on both sides.

For the treatment, the modified Epley method was used in the case of canalolithiasis of the posterior semicircular canal, the Barbecue rotation method was used in the case of canalolithiasis of the lateral semicircular canal, and the Appiani and Gufoni methods were used in the case of cupulolithiasis. The treatment was performed with a particle repositioning maneuver once a day with intervals of 2 to 3 days. The criteria for a complete cure were determined to be the disappearance of symptoms and the disappearance of nystagmus in the physical examinations. The criterion for recurrence was a diagnosis of BPPV based on the confirmation of nystagmus with video Frenzel goggles due to dizziness that occurred again at least one month after the complete cure.

The median (interquartile range, IQR), frequency, and ratio values were used for the descriptive statistics of the data, and the parametric quantitative data were compared using the Student $t$ test. The parametric frequency data were compared using the Chi-squared test. The non-parametric Kruskal-Wallis test for $\mathrm{k}$ independent samples was mostly used when there were non-parametric data. Values of $p<0.05$ indicated statistical significance. The Statistical Package for the Social Sciences (SPSS, IBM Corp., Armonk,
NY, US) software, version 19.0, was used for the statistical analysis.

\section{Results}

There were 25 patients ( 4 males and 21 females) in the case group, and their median age was 40 years. The control group consisted of 50 patients ( 8 males and 42 females; median age: 45 years; $p<0.079$ ) selected by $1: 2$ frequency matching using age, gender, and canal types of BPPV as matching variables. In the case group, 14 patients had canalolithiasis (posterior canal in 1 patient, lateral canal in 9 patients, and multiple canals in 4 patients), and 11 patients had cupulolithiasis. The control group consisted of 26 patients with canalolithiasis (posterior canal in 3 patients, lateral canal in 18 patients, and multiple canals in 5 patients), and 24 patients with cupulolithiasis.

During the follow-up period of 24 months, among the patients in the case group, 3 had 1 relapse and 4 had 2 relapses, and among the patients in the control group, 9 had 1 relapse, 2 had 2 relapses, and 2 had 3 relapses $(p<0.311)$. The total number of relapses in the case and control groups was 10 and 18 respectively $(p<0.243)$. The $25-\mathrm{OH}$ vitamin D concentrations were of $6.06 \mathrm{ng} / \mathrm{mL}$ in the case group, and of $7.14 \mathrm{ng} / \mathrm{mL}$ in the control group at the beginning of the experiment $(p<0.792)$. The $25-\mathrm{OH}$ vitamin D concentration in the case group was of $31.1 \mathrm{ng} \mathrm{mL}$ at 1 year of follow-up. There were 2 hypertensive patients in the case group, and 13 in the control group $(p<0.076)$; regarding diabetes there

Table 1 Characteristics of the case and control groups

\begin{tabular}{|l|l|l|l|}
\hline & $\begin{array}{l}\text { Case } \\
\text { group } \\
(\boldsymbol{n}=\mathbf{2 5})\end{array}$ & $\begin{array}{l}\text { Control } \\
\text { group } \\
(\boldsymbol{n}=\mathbf{5 0 )}\end{array}$ & $p$-value \\
\hline Gender (male;female) & $4 ; 21$ & $8 ; 42$ & \\
\hline $\begin{array}{l}\text { Age: years (interquartile } \\
\text { range, IQR) }\end{array}$ & $40(13.5)$ & $45(23.75)$ & 0.079 \\
\hline $\begin{array}{l}\text { Number of subjects } \\
\text { with recurrence } \\
\text { (single;two;three) }\end{array}$ & \begin{tabular}{l}
$(3 ; 4 ; 0)$ \\
\hline $\begin{array}{l}\text { Total number of } \\
\text { recurrence attacks }\end{array}$
\end{tabular} & $\begin{array}{l}13 \\
(9 ; 2 ; 2)\end{array}$ & 0.311 \\
\hline $\begin{array}{l}\text { Type of benign } \\
\text { paroxysmal } \\
\text { positional vertigo: } \\
\text { canalolithiasis } \\
\text { (posterior canal;lateral } \\
\text { canal;multiple canals) } \\
\text { cupulolithiasis }\end{array}$ & $\begin{array}{l}14 \\
(1 ; 9 ; 4)\end{array}$ & $\begin{array}{l}(3 ; 18 ; 5) \\
24\end{array}$ & 0.243 \\
\hline $\begin{array}{l}\text { 25-hydroxyvitamin D } \\
\text { before treatment (IQR) }\end{array}$ & $6.06(3.5)$ & $7.14(3.0)$ & 0.792 \\
\hline $\begin{array}{l}\text { 25-hydroxyvitamin D } \\
\text { after treatment (IQR) }\end{array}$ & $31.1(6.8)$ & 2 & 0.256 \\
\hline Hypertension & 2 & 13 & 0.550 \\
\hline Diabetes & 0 & 7 & 0.076 \\
\hline Hyperlipidemia & 1 & 26 & \\
\hline
\end{tabular}

Notes: Result of t-test are age and vitamin D. The others are Chi-squared test. No significant differences were detected between the subgroups. 
Table 2 Effect of vitamin D injection on benign paroxysmal positional vertigo patients in the case and control groups

\begin{tabular}{|c|c|c|c|c|c|c|c|c|c|}
\hline & \multirow[t]{2}{*}{ Follow-up period } & \multicolumn{2}{|l|}{ 0-6 months } & \multicolumn{2}{|c|}{$7-12$ months } & \multicolumn{2}{|c|}{ 13-24 months } & \multicolumn{2}{|l|}{ Total } \\
\hline & & Recurrence & $\begin{array}{l}\text { Exact } \\
\text { sig. }\end{array}$ & Recurrence & $\begin{array}{l}\text { Exact } \\
\text { sig. }\end{array}$ & Recurrence & $\begin{array}{l}\text { Exact } \\
\text { sig. }\end{array}$ & Recurrence* & $\begin{array}{l}\text { Exact } \\
\text { sig. }\end{array}$ \\
\hline \multirow{2}{*}{$\begin{array}{l}\text { Subgroups } \\
\text { I,III }\end{array}$} & Case $(n=10)$ & 2 & \multirow[t]{2}{*}{1.000} & 1 & \multirow[t]{2}{*}{0.323} & 0 & \multirow[t]{2}{*}{1.000} & 2 & \multirow[t]{2}{*}{0.756} \\
\hline & Control $(n=21)$ & 3 & & 0 & & 1 & & 4 & \\
\hline $\begin{array}{l}\text { Subgroups } \\
\text { II,IV }\end{array}$ & $\begin{array}{l}\text { Case }(n=15) \\
\text { Control }(n=29)\end{array}$ & $\begin{array}{l}4 \\
5\end{array}$ & 0.695 & $\begin{array}{l}1 \\
4\end{array}$ & 0.647 & $\begin{array}{l}2 \\
5\end{array}$ & 1.000 & $\begin{array}{l}5 \\
9\end{array}$ & 0.998 \\
\hline \multirow[t]{2}{*}{ Total } & \multirow{2}{*}{$\begin{array}{l}\text { Case }(n=25) \\
\text { Control }(n=50)\end{array}$} & 6 & \multirow[t]{2}{*}{0.531} & 2 & \multirow[t]{2}{*}{1.000} & 2 & \multirow[t]{2}{*}{0.711} & 7 & \multirow[t]{2}{*}{0.883} \\
\hline & & 8 & & 4 & & 6 & & 13 & \\
\hline
\end{tabular}

Abbreviation: Exact sig, Exact test of significance.

Notes: Non-parametric Kruskal-Wallis test for k independent samples. Subgroups I,III: posterior canal and lateral canal benign paroxysmal positional vertigo. Subgroups II,IV: cupulolithiasis and multiple canal benign paroxysmal positional vertigo. *One patient's recurrence was counted as once.

were 0 cases in the case group and 2 in the control group $(p<0.256)$; and there was 1 hyperlipidemic patient in the case group and 7 in the control group $(p<0.550)$ ( - Table 1 ).

As shown in - Table $\mathbf{2}$, the differences in relapse rates between the case and control groups were examined using the non-parametric Kruskal-Wallis test for $\mathrm{k}$ independent samples. The differences in relapse rates between subgroups I (10 patients in the case group) and III ( 21 patients in the control group) were compared by period: from 0 to 6 months, 7 to 12 months, 13 to 24 months, and over the entire period. There was no statistically significant difference between the 2 groups after 6 months because 2 patients in the case group and 3 patients in the control group had relapses, with $p<1.000$. In the period of 7 to 12 months, 1 patient in the case group and 0 in the control group had relapses $(p<0.323)$; in the period of 13 to 24 months, 0 patients in the case group and 1 in the control group had relapses $(p<1.000)$; and, during the entire period, 2 patients in the case group and 4 in the control group had relapses, with $p<0.756$ indicating that there was no statistical significance.

The relapse rates of subgroups II (15 patients in the case group) and IV (29 patients in the control group) were compared. Within 6 months, 4 patients in the case group and 5 in the control group had relapses, with $p<0.695$ indicating that there was no statistical significance. In the period of 7 to 12 months, 1 patient in the case group and 4 in the control group had relapses ( $p<0.647$ ); in the period of 13 to 24 months, 2 patients in the case group and 5 in the control group had relapses ( $p<1.000)$; and, during the entire period, 5 patients in the case group and 9 in the control group had relapses, with $p<0.998$ indicating that there was no statistical significance. With regard to the relapse rates of the entire case and control groups by period, within 6 months, 6 patients in the case group and 8 in the control group had relapses, with $p<0.531$ indicating that there was no statistical significance. In the period of 7 to 12 months, 2 patients in the case group and 4 in the control group had relapses $(p<1.000)$; in the period of 13 to 24 months, 2 patients in the case group and 6 in the control group had relapses $(p<0.711)$; and, during the entire period, 7 patients in the case group and 13 in the control group had relapses, with $p<0.883$ indicating that there was no statistical significance (-Table 2).

\section{Discussion}

There are case reports and other studies indicating that the episodes of recurrence of BPPV decreased when the patients took oral vitamin D every time they had a vitamin D deficiency. ${ }^{13,14}$ In the present study, vitamin D injection therapy was implemented to reduce recurrences occurring within twentyfour months after the onset of BPPV, during which the risk of recurrence is the highest, and case-control studies were conducted. A decrease in the number of recurrences after implementing vitamin $\mathrm{D}$ therapy in severe recurrence patients was also observed by the author of the present study. This experience was the reason to conduct the study on vitamin D injection therapy. However, the results of the follow-ups for twenty-four months showed no significant difference between the vitamin D injection group and the control group, which went against the author's expectations.

The long-term recurrence rate of BPPV is generally known to reach $40 \%$ to $50 \%$ between 3 and 5 years. ${ }^{2,3}$ However, the results of one study ${ }^{14}$ indicate that most recurrences occur within one year, especially within six months after the first episode. Age, accompanying otologic disease, the female gender, type of canal, number of particle repositioning maneuvers, chronic disease, and vitamin $\mathrm{D}$ deficiency are known as factors associated with recurrences. ${ }^{2,3,15}$ Among them, the strongest factor is age. According to von Brevern et $\mathrm{al}^{16}{ }^{16}$ the cumulative prevalence of BPPV begins to increase rapidly from age 40 . In addition, the prevalence of BPPV among females is twice as high as the prevalence among males. This difference is often explained by differences in sex hormones. Therefore, studies on the association between osteoporosis and BPPV have also been mostly conducted with postmenopausal women with rapid changes in sex hormones. ${ }^{8,9}$ Vitamin $\mathrm{D}$ is an important factor in calcium metabolism, and is a well-known factor associated with bone metabolic diseases such as osteoporosis. Some studies indicated that vitamin D deficiency increases the risk of fracture, and vitamin D supplementation therapy reduces the risk of fracture in elderly patients. ${ }^{17,18}$ In otoconia 
metabolism studies ${ }^{5,12}$ in relation to BPPV, the results indicated that vitamin $\mathrm{D}$ is involved in the calcium metabolic process, and that vitamin D deficiency affects the occurrence of BPPV. But there are studies ${ }^{19,20}$ that show no relationship between vitamin D deficiency and BPPV. According to Gülşah et al, ${ }^{19}$ no relationship was found between vitamin $D$, total calcium levels, BPPV incidence and recurrence. And a meta-analysis ${ }^{21}$ by AlGarni et al failed to establish a relationship between the occurrence of BPPV and low vitamin D levels.

However, vitamin D supplementation therapy has recently been attracting attention in an effort to reduce the recurrence of BPPV. Vitamin D injection therapy has the effects of more rapidly normalizing blood concentrations than oral therapy, and of maintaining the drug concentration constant during the treatment period. In addition, the maintained concentration lasts longer, and there is not the inconvenience of taking the vitamin every day as with the oral therapy. Therefore, the reliability and easiness of the injection therapy can be said to be higher than those of the oral therapy. In another study, ${ }^{22}$ after an intramuscular injection of 150,000-250,000 IU of vitamin $\mathrm{D}_{3}$, the blood concentration of vitamin D was maintained at $20-25 \mathrm{ng} / \mathrm{mL}$ on average 12 months later. In a study conducted by Choi et $\mathrm{al}^{23}$ with South Koreans, 93\% of the patients maintained blood concentrations of vitamin $\mathrm{D} \geq 20 \mathrm{ng} / \mathrm{mL} 3$ months after the injection, and $96 \%$ of the patients maintained concentrations $\geq 20 \mathrm{ng} / \mathrm{mL} 6$ months after the injection. No side effect was reported during the follow-up period.

The determination of the concentration levels of 25-OH vitamin $\mathrm{D}$ for the treatment is important. This is still under discussion in the case of vitamin D therapy for osteoporosis. Although most studies concluded that vitamin D therapy is effective in the treatment of patients with osteoporosis with vitamin D deficiency, the results of a randomized, doubleblinded, placebo-controlled trial on the relationships between vitamin $\mathrm{D}$ and bone density and muscle functions conducted with patients with vitamin D blood concentrations $\geq 21 \mathrm{ng} / \mathrm{mL}$ by Hasen et $\mathrm{al}^{24}$ indicated that there were no differences between the group submitted to vitamin D therapy and the control group regarding the effects of the treatment.

In the present study, the target point of therapeutic concentration of $25-\mathrm{OH}$ vitamin $\mathrm{D}$ was set as $\geq 20 \mathrm{ng} / \mathrm{mL}$, and the blood concentration of vitamin $\mathrm{D}$ in the case group for the first year of follow-up was of $31.1 \mathrm{ng} / \mathrm{mL}$.

The reasons why there was no difference in the rate of recurrence between the vitamin $\mathrm{D}$ injection group and the control group can be assumed as follows: first, the vitamin $\mathrm{D}$ injection may not have an immediate therapeutic effect. In general, the vitamin $\mathrm{D}$ blood concentration recovers to at least $20 \mathrm{ng} / \mathrm{mL}$ within 2 weeks after vitamin D injections. However, since the result of a long-term follow-up study ${ }^{17}$ on osteoporosis indicated that the total body bone mineral density began to recover only one year after the injection, the normalization of calcium metabolism that is associated with otoconia in BPPV may also take a long time.

Second, it is assumed that, although vitamin $\mathrm{D}$ acts as a factor associated with BPPV recurrence, it may not be effective as a therapeutic agent because its role is not important.
However, according to Talaat et $\mathrm{al}^{14}{ }^{14}$ the recurrence rate decreased after 18 months of follow-up after vitamin D therapy in patients with BPPV limited to the posterior semicircular canal. Given the study ${ }^{2,15}$ results that indicate that recurrence rates change depending on the type of canal affected (the lateral canal or multiple canals), vitamin D supplementation therapy may be effective under limited conditions. The limitations of our study are as follows: the treatment effects of vitamin D are not yet clear, and may vary depending on the type of canal affected. In the present study, there were many cases of lateral canalolithiasis and cupulolithiasis rather than the posterior canal type, making it difficult to compare with previous studies.

However, because there are many factors associated with $B P P V$ recurrence, for vitamin $D$ to be a strong and effective therapeutic factor regarding BPPV recurrence in vitamin D deficient patients, case-control studies that are larger than the present study are necessary.

Among the recent studies on the relationship between metabolic diseases and BPPV, some studies indicate that recurrences increase along with the number of chronic diseases, such as diabetes and hypertension, ${ }^{25}$ and other studies $^{15,26}$ indicate that hypertension and hyperlipidemia are factors associated with BPPV.

Vitamin D deficiency is known to increase the risk of metabolic syndrome by increasing insulin resistance, and vitamin D supplementation is known to be helpful in the treatment of hypertension by lowering systolic blood pressure. ${ }^{18}$ Eventually, vitamin D deficiency is thought to act on metabolic diseases, thereby directly or indirectly affecting the recurrence of BPPV. However, in the present study, the diabetes, hypertension, and hyperlipidemia of the vitamin D deficient patients did not affect BPPV recurrences.

\section{Conclusion}

The present case-control study indicated that vitamin $D_{3}$ injection had no significant effect on the recurrences of BPPV in patients with vitamin $\mathrm{D}$ deficiency when age, gender, and type of BPPV were homogeneous between the two groups.

\section{Conflict of Interests}

The authors have no conflict of interests to declare.

\section{References}

1 Tanimoto H, Doi K, Nishikawa T, Nibu K. Risk factors for recurrence of benign paroxysmal positional vertigo. J Otolaryngol Head Neck Surg 2008;37(06):832-835

2 Sakaida M, Takeuchi K, Ishinaga H, Adachi M, Majima Y. Long-term outcome of benign paroxysmal positional vertigo. Neurology 2003;60(09):1532-1534

3 Brandt T, Huppert D, Hecht J, Karch C, Strupp M. Benign paroxysmal positioning vertigo: a long-term follow-up (6-17 years) of 125 patients. Acta Otolaryngol 2006;126(02):160-163

4 Lundberg YW, Xu Y, Thiessen KD, Kramer KL. Mechanisms of otoconia and otolith development. Dev Dyn 2015;244(03): 239-253

5 Rhim GI. Serum vitamin D and recurrent benign paroxysmal positional vertigo. Laryngoscope Investig Otolaryngol 2016;1 (06):150-153 
6 Xu Y, Zhang H, Yang H, Zhao X, Lovas S, Lundberg YW. Expression, functional, and structural analysis of proteins critical for otoconia development. Dev Dyn 2010;239(10):2659-2673

7 Lundberg YW, Zhao X, Yamoah EN. Assembly of the otoconia complex to the macular sensory epithelium of the vestibule. Brain Res 2006;1091(01):47-57

8 Vibert D, Kompis M, Häusler R. Benign paroxysmal positional vertigo in older women may be related to osteoporosis and osteopenia. Ann Otol Rhinol Laryngol 2003;112(10):885-889

9 Yamanaka T, Shirota S, Sawai Y, Murai T, Fujita N, Hosoi H. Osteoporosis as a risk factor for the recurrence of benign paroxysmal positional vertigo. Laryngoscope 2013;123(11):2813-2816

10 Jeong SH, Choi SH, Kim JY, Koo JW, Kim HJ, Kim JS. Osteopenia and osteoporosis in idiopathic benign positional vertigo. Neurology 2009;72(12):1069-1076

11 Jeong SH, Kim JS, Shin JW, et al. Decreased serum vitamin D in idiopathic benign paroxysmal positional vertigo. J Neurol 2013; 260(03):832-838

12 Talaat HS, Abuhadied G, Talaat AS, Abdelaal MSS. Low bone mineral density and vitamin $D$ deficiency in patients with benign positional paroxysmal vertigo. Eur Arch Otorhinolaryngol 2015; 272(09):2249-2253

13 Büki B, Ecker M, Jünger H, Lundberg YW. Vitamin D deficiency and benign paroxysmal positioning vertigo. Med Hypotheses 2013;80 (02):201-204

14 Talaat HS, Kabel AMH, Khaliel LH, Abuhadied G, El-Naga HAER, Talaat AS. Reduction of recurrence rate of benign paroxysmal positional vertigo by treatment of severe vitamin $\mathrm{D}$ deficiency. Auris Nasus Larynx 2016;43(03):237-241

15 Pérez P, Franco V, Cuesta P, Aldama P, Alvarez MJ, Méndez JC. Recurrence of benign paroxysmal positional vertigo. Otol Neurotol 2012;33(03):437-443

16 von Brevern M, Radtke A, Lezius F, et al. Epidemiology of benign paroxysmal positional vertigo: a population based study. J Neurol Neurosurg Psychiatry 2007;78(07):710-715
17 Dawson-Hughes B, Harris SS, Krall EA, Dallal GE. Effect of calcium and vitamin D supplementation on bone density in men and women 65 years of age or older. N Engl J Med 1997;337(10): 670-676

18 Mosekilde L. Vitamin D and the elderly. Clin Endocrinol (Oxf) 2005;62(03):265-281

19 Gülşah ÇI, Yunsur Ç, Emine E, Şeref KÇ. Analysis of Vitamin D and Calcium Levels in Benign Paroxysmal Positional Vertigo. Eurasian J Emerg Med 2017;16:128-132

20 Karataş A, Acar Yüceant G, Yüce T, Hacı C, Cebi IT, Salviz M. Association of Benign Paroxysmal Positional Vertigo with Osteoporosis and Vitamin D Deficiency: A Case Controlled Study. J Int Adv Otol 2017;13(02):259-265

21 AlGarni MA, Mirza AA, Althobaiti AA, Al-Nemari HH, Bakhsh LS Association of benign paroxysmal positional vertigo with vitamin D deficiency: a systematic review and meta-analysis. Eur Arch Otorhinolaryngol 2018;275(11):2705-2711

22 Vieth R. Vitamin D supplementation, 25-hydroxyvitamin D concentrations, and safety. Am J Clin Nutr 1999;69(05): 842-856

23 Choi HS, Chung YS, Choi YJ, Seo DH, Lim SK. Efficacy and safety of vitamin $\mathrm{D}_{3}$ B.O.N intramuscular injection in Korean adults with vitamin D deficiency. Osteoporos Sarcopenia 2016;2(04): 228-237

24 Hansen KE, Erin Johnson R, Chambers KR, et al. Treatment of vitamin $\mathrm{D}$ insufficiency in postmenopausal women: a randomized clinical trial. JAMA Intern Med 2015;175(10): 1612-1621

25 De Stefano A, Dispenza F, Suarez H, et al. A multicenter observational study on the role of comorbidities in the recurrent episodes of benign paroxysmal positional vertigo. Auris Nasus Larynx 2014;41(01):31-36

26 Ogun OA, Janky KL, Cohn ES, Büki B, Lundberg YW. Gender-based comorbidity in benign paroxysmal positional vertigo. PLoS One 2014;9(09):e105546 